\title{
S/TEM Observation of Catalyst Growth on a Si Substrate
}

\author{
Michael Larsen, John Osaheni and Keith Borst
}

General Electric Global Research Center, Niskayuna, NY 12309

The growth of carbon nanotubes on a silicon substrate is aided by the presence of catalyst particles. The composition of the particles as well as their size, size distribution and spatial distribution on the silicon substrate is important in determining the final carbon nanotube size and distribution. As an example, the diameter of the catalyst particles will determine the diameter of the carbon nanotubes. Though the importance of catalyst particles on the growth of carbon nanotubes is clear, the mechanism of how the catalyst form on the substrate is less clear. The following study was undertaken to gain some insight into this mechanism.

In order to facilitate the $\mathrm{S} / \mathrm{TEM}$ investigation, silicon wafers were first made into TEM "foils". This was done by core drilling out $3 \mathrm{~mm}$ diameter disks of silicon from a silicon wafer. The disks were then mechanically ground to a thickness of about 100um. The thinned disk was dimpled and ion milled until perforation. This TEM ready disk was then used as the substrate for the catalyst particles. A Tecnai F20 S/TEM, which is equipped with an Oxford INCA EDS unit, was used for the study.

Figure 1a shows the bright field TEM image of material sputtered onto a TEM ready silicon substrate from an alloy target. The target had a nominal composition 52Co-26Al-22Cr (atomic \%). The material was sputtered for 10 seconds at $500 \mathrm{~V}$ and 200 watts with Ar gas flowing at $9 \mathrm{ml}$ per minute. The material is present on the Si substrate as a nearly continuous amorphous film. Elemental analysis by EDS of this as-deposited material show peaks from $\mathrm{Cr}$ and $\mathrm{Co}$ (figure $1 \mathrm{~b}$ ). A small Al peak may be present, but is difficult to distinguish from the large Si peak. .

The result of heating the sample to $750 \mathrm{C}$ for 5 minutes in an inert environment $(1000 \mathrm{sccm}$ argon flow) is shown in figure 2. The bright field TEM images seen in figure 2a show a high density of particles with two distinct morphologies. There are small, spherical particles with a range of diameters all less than 10nm. There are also larger rod-like (or possibly disk shaped) particles, which are about $20 \mathrm{~nm}$ long, and $10 \mathrm{~nm}$ wide. The larger particles have an orientation relationship with the underlying silicon substrate. When the substrate is oriented in the (001) zone axis as is the case in figure 2, two variants of the larger rod-like particles are readily visible. The long axis of these two variants lies in the direction of two of the substrates cube axis. A third variant, corresponding to the third cube axis is most likely present, though difficult to see in this image.

The nature of these particles was further investigated using EDS in the STEM mode. Figure $2 \mathrm{~b}$ shows a STEM image of the annealed sample. EDS from the particles shows that the smaller spherical particles are primarily $\mathrm{Cr}$ with small amount of $\mathrm{Co}$ (figure $2 \mathrm{c}$ ). The larger rod-like particles are primarily Co (figure $2 \mathrm{~d}$ ). A spectrum taken between particles shows only Si from the substrate (figure 2e).

This study has shown that the growth of catalyst particles on a Si substrate can be complex. When the sputtering target is an alloy, not all of the elements in alloy necessarily end up on the substrate. In our case, very little if any $\mathrm{Al}$ is present on the substrate though the sputtering target contained a high level of Al. Also, sputtering from a single target can result in the growth of more than one type of particle. A systematic approach to growing carbon nanotubes on Si substrates with the aid of catalyst particles will need to take these complications into account. 


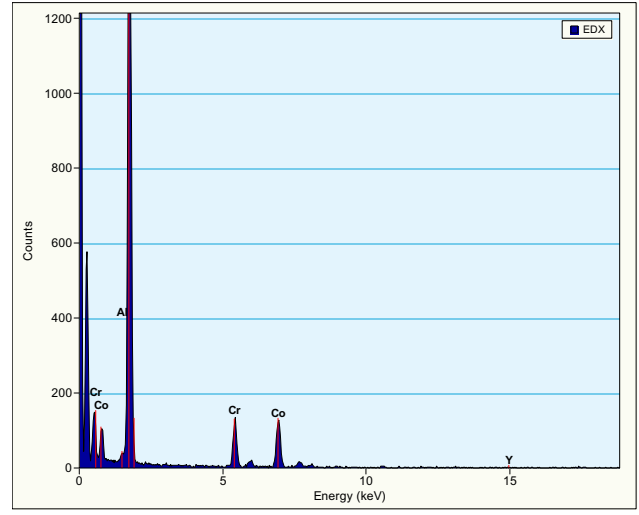

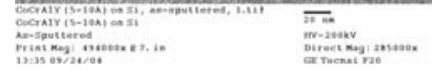

(1a) (1b)

Figure1. As-sputtered 52Co26A122Cr on $\mathrm{Si}$. EDS shows that it is primarily $\mathrm{Co}$ and $\mathrm{Cr}$.

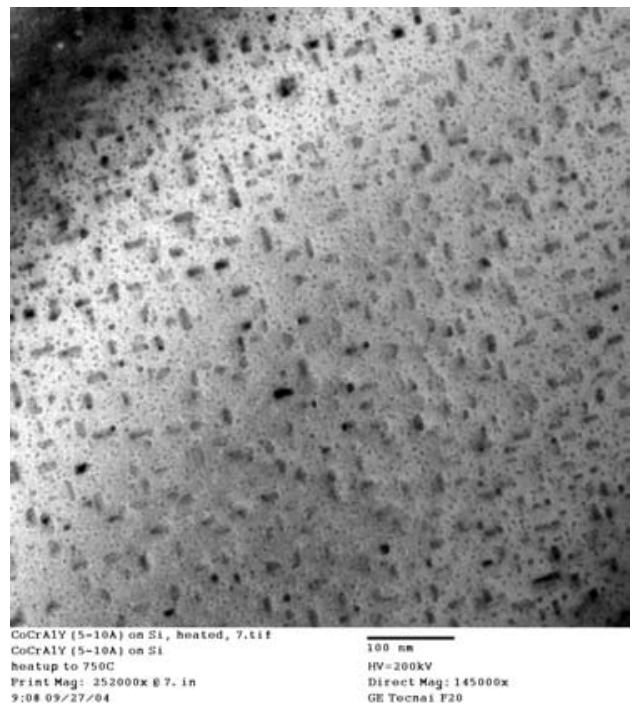

(2a)

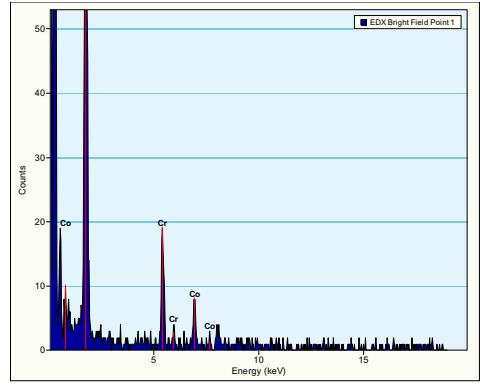

$(2 \mathrm{c})$

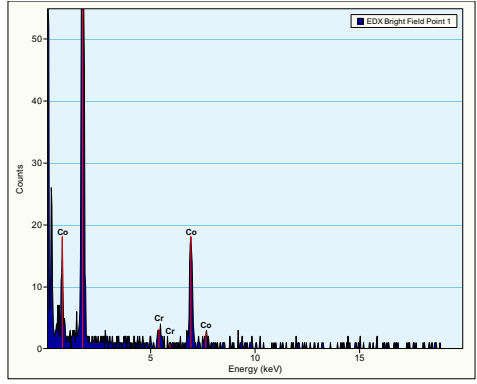

$(2 \mathrm{~d})$

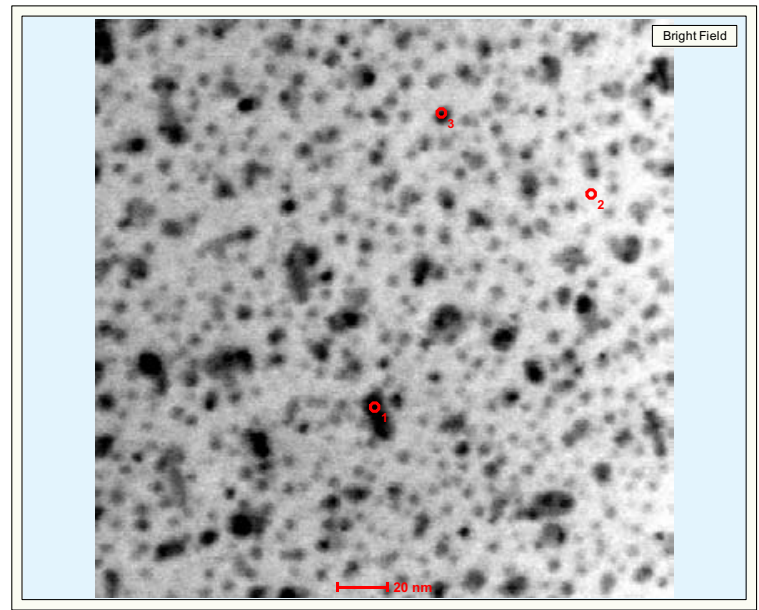

$(2 b)$

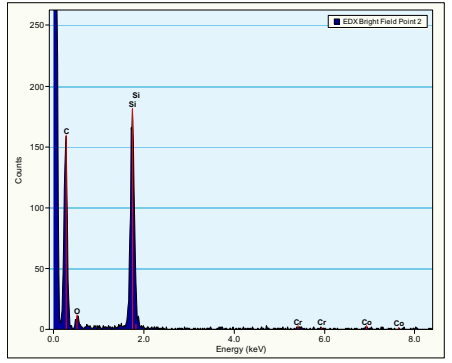

$(2 \mathrm{e})$

Figure 2. 52Co26A122 $\mathrm{Cr}$ sputtered on $\mathrm{Si}$, annealed 750C, 5sec. Two particles types are present. Bright field image (2a). STEM image of annealed sample (2b). EDS shows that the small, spherical particles are $\mathrm{Cr}$ rich (2c). The larger elongated particles are Co rich (2d). Only $\mathrm{Si}$ is present between particles (2e). 